\title{
Magnetic excitations in assemblies of dipolar coupled nanoparticles
}

\author{
Fabrice Boust $^{1, *}$ and Nicolas Vukadinovic ${ }^{2, * *}$ \\ ${ }^{1}$ DEMR, ONERA, Université Paris-Saclay, F-91123, Palaiseau, France \\ ${ }^{2}$ Dassault Aviation, 78 quai Marcel Dassault, 92552 St-Cloud, France
}

\begin{abstract}
The equilibrium magnetization configurations and the associated microwave susceptibility spectra of dipolar coupled nanoplatelets are explored using three-dimensional (3D) micromagnetic simulations. First, the case of periodic arrangements of nanoplatelets on square arrays is considered. As a result, a macro-vortex state defined as a flux closure pattern spreading over the whole array with or without a vortex core can be stabilized starting from an initial orthoradial magnetization configuration. For macro-vortex states with a vortex core, the linear excitation spectrum exhibits a sub-GHz resonance line ascribed to the vortex core dynamics at the array center. The features of this line (spectral position and amplitude) depend on the array size and the strength of the dipolar coupling through the interplatelet spacing. This resonance is also observed for macro-vortex states without a vortex core but only in the regime of a strong dipolar coupling. The effect of disorder is then investigated by numerically generating assemblies of nanoplatelets with a position disorder and, shape and size distributions. The micromagnetic simulations reveal flux closure magnetization configurations as well but without a vortex core. A low-frequency resonance appears in the susceptibility spectra for quite high surface contents of nanoplatelets but its amplitude is weaker compared to the case of periodic arrays. This line arises from a collective mode extended over a few nanoplatelets.

A large variety of static and dynamical behaviors is thus evidenced resulting in a great complexity even in such model systems.
\end{abstract}

\section{Introduction}

Magnetic nanoobjects are the building blocks shared by a wide range of promizing technologies like the future ultrahigh density storage media [1], the nanomagnet-based logic functions for new data processing systems [2], the energy-harvesting devices [3], the biomedical applications [4] and the miniaturized microwave components [5]. Design of new devices integrating such nanoobjects requires a deep understanding of their static and dynamical magnetic properties both as isolated and interacting objects. Schematically, the assemblies of nanoobjects can be classified in two categories, namely, the geometrically ordered ones and the disordered ones. The ordered systems correspond to periodic arrangements of nanoobjects [6]. The artificial spin ices defined as a two-dimensional arrays of closed spaced single-domain nanoobjects coupled by dipolar interaction belong to this family [7]. One remarkable feature of such systems is the appearence of a magnetic frustration due to the impossibility of satisfying simultaneously all competing dipolar interactions between the nanoobjects. The static and dynamical properties of artificial spin ices are controlled by the shape, the size and the composition of the nanoobjects, and the geometrical properties of arrays (symmetry and lattice constants). A large variety of equilibrium magnetization configurations has been experimentally and numerically reported [7, 8].

\footnotetext{
*e-mail: fabrice.boust@onera.fr

**e-mail: nicolas.vukadinovic@dassault-aviation.com
}

In addition, the excitation spectrum at microwave frequencies experimentally and numerically investigated reveals the presence of collective spin-wave modes [9] and reflects the existence of topological defects within the array [10]. On the other hand, disordered assemblies of magnetic nanoobjects are exemplified by nanocomposites where ferromagnetic nanoparticles are embedded in a nonmagnetic matrix [11]. In contrast to ordered systems, the numerical simulations based on the micromagnetism theory for describing the dynamic response of such systems are rather rare. The effective susceptibility spectra of the nanocomposites are usually computed using phenomenological approaches based on mixing rules [12]. Even if such models can incorporante some information regarding the composite morphology like the existence of nanoparticles clusters [11], this approach suffers from severe approximations. In particular, the particles are assumed to be in the single-domain state and the dipolar interaction between the particules is treated by means of an effective demagnetizing factor [11].

The aim of this paper is to investigate the equilibrium magnetization configurations and the associated microwave excitation spectra in the linear regime of dipolar coupled nanoplatelets by means of three-dimensional (3D) micromagnetic simulations. The characteristics of nanoplatelets have been chosen to allow the occurence of nonuniform magnetization states within the individual elements. First, the effect of the dipolar coupling strength on the equi- 
librium micromagnetic configurations and the susceptiblity spectra is studied in the case of periodic arrays of nanoplatelets. Then, aggregates of randomly distributed nanoplatelets are considered and the impact of geometrical disorder on the microwave response is analyzed. Emphasis is placed on the existence of a sub-GHz resonance resulting from the dynamics of flux closure magnetization configurations.

\section{Numerical model}

\subsection{Generation of the nanoparticles arrangements}

(a)

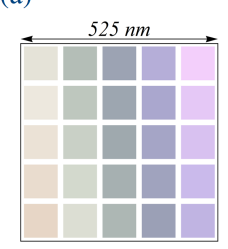

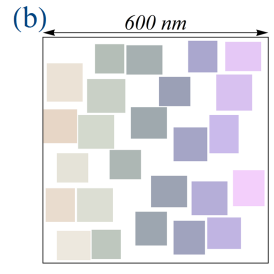

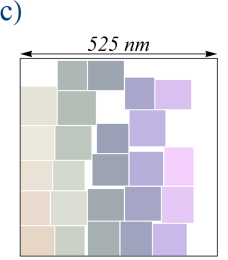

Figure 1. Examples of 25 nanoplatelets assemblies numerically generated. (a) Ordered assembly on a periodic square array. Disordered assemblies with a surface content $\varphi_{s}=56 \%$ (b) and $\varphi_{s}=81 \%$ (c). The color code is only for esthetic purpose.

The nominal nanoelements of interest are thin squareshaped platelets defined by the platelet side $\mathrm{L}$ and and the platelet thickness $L_{z}$, the z-axis being along the platelet thickness. In the conducted numerical simulations, $L=90$ nm and two values of $L_{z}$ were considered: $L_{z}=4 \mathrm{~nm}$ and $L_{z}=12 \mathrm{~nm}$.

For the ordered assemblies, the nanoplatelets were arranged in two-dimensional square arrays. The interplatelet spacing $\delta$ (edge-to-edge distance) was varied between 3 $\mathrm{nm}$ and $30 \mathrm{~nm}$. $\delta$ is related to the lattice constant $a$ by: $a=L+\delta$. Various array sizes were investigated: $N=2 \times 2$, $3 \times 3,4 \times 4,5 \times 5$, and $6 \times 6$, where $\mathrm{N}$ is the number of periodic cells equal to the number of platelets. Figure 1(a) shows an example of a $5 \times 5$ array of nanoplatelets with $\delta=15 \mathrm{~nm}$.

For the disordered assemblies, the degree of disorder was introduced using the following two-step generation rules. First, the nominal square-shaped nanoplatelets can be transformed into rectangular ones with the in-plane sizes $L_{x}$ and $L_{y}$ inside the size range $80 \mathrm{~nm}<L_{x}, L_{y}<100 \mathrm{~nm}$. Second, the position of the nanoplatelets can be changed from the initial one in the square array by applying spatial deviations $\delta_{x}$ and $\delta_{y}$ along the $\mathrm{x}$ and y axes, respectively. These deviations satisfy $4 \mathrm{~nm}<\delta_{x}, \delta_{y}<12 \mathrm{~nm}$ and the excluded volume rule (no overlap between the platelets). In both cases, $L_{x}, L_{y}, \delta_{x}$ and $\delta_{y}$ result from a random draw with a uniform distribution. Each configuration is characterized by the surface content of nanoplatelets defined as $\varphi_{s}=N L_{x} L_{y} / S$, where $\mathrm{S}$ is the surface of the resized arrays. Figures 1(b) and 1(c) display two examples of disordered configurations with $\varphi_{s}=56 \%$ and $\varphi_{s}=81 \%$, respectively.

\subsection{Micromagnetic simulations}

The high-frequency response of ordered and disordered nanoplatelets assemblies was computed by means of micromagnetic simulations using two 3D home made codes described elsewhere [13]. The first code computes an equilibrium magnetization configuration by integrating the Landau-Lifshitz (LL) equation in the time domain using a second-order Taylor scheme and an optimized time step. The second one solves the LL equation linearized around the equilibrium magnetization configuration (small-amplitude motion regime) in the frequency domain. It computes the local dynamic susceptibility tensor $\chi_{i j}(\mathbf{r}, \omega) \quad i, j=x, y, z$ which depends both on space and angular frequency, and then the dynamic susceptibility tensor averaged over the element's volume V, $\chi_{i j}(\omega)=\left\langle\chi_{i j}(\mathbf{r}, \omega)\right\rangle_{V} i, j=x, y, z$, which connects the high-frequency response of a magnetic configuration $\delta \mathbf{m}$ to a weak exciting RF magnetic field $\delta \mathbf{h}$ such as $\delta \mathbf{m}=$ $\bar{\chi} \delta \mathbf{h}$. These codes were previously used to investigate the microwave response of vortex-state [14] and bubblestate [15] single elements. The micromagnetic simulations were performed using magnetic parameters representative of isotropic Permalloy $\left(N i_{80} F e_{20}\right)$ for each nanoplatelet, namely : the saturation magnetization $M_{S}=8.10^{5} \mathrm{~A} / \mathrm{m}$, the exchange constant $A=1.310^{-11} \mathrm{~J} / \mathrm{m}$, the gyromagnetic ratio $\gamma=1.7610^{11} \mathrm{~s}^{-1} T^{-1}$ and the damping parameter $\alpha=0.02$. The nanoplatelets are spatially discretized using a regular cubic mesh. The mesh sizes were chosen to be equal to $\Delta_{x}=\Delta_{y}=\Delta_{z}=3 \mathrm{~nm}$ lower than the exchange length, $\left.\Lambda=\sqrt{2 A /\left(\mu_{0} M_{s}^{2}\right.}\right) \simeq 5.7 \mathrm{~nm}$.

\section{Numerical results}

\subsection{Isolated nanoplatelets}

Various stable magnetization configurations exist in soft planar magnetic nanostructures depending on the element size and thickness [16]. Let us consider the case of isolated square-shaped platelets with $L=90 \mathrm{~nm}$ and $L_{z}=12$ $\mathrm{nm}$. Three equilibrium magnetization configurations can be stabilized in such platelets. The first configuration is the vortex state described as an in-plane curling magnetization with a central region, the vortex core, where the magnetization points out of plane (Fig. 2(a)). The magnetic energy of this vortex state is equal to $26.6 \mathrm{ev}$. In what follows, the magnetic energy of various magnetic configurations will be normalized by the vortex one. The second configuration is the onion state (also termed leaf state) where the magnetization has a privileged in-plane orientation along one diagonal of the square platelet (Fig. 2(b)). The reduced magnetic energy is $\epsilon_{o}=0.945$. The third magnetic configuration is the so-called buckle or $\mathrm{C}$ state where the in-plane magnetization adopts a C-like shape (Fig. 2(c)). For this case, $\epsilon_{c}=0.942$. These three stable states have magnetic energies close to each other with the $\mathrm{C}$ state being the lowest-energy state. Figure 2(h) displays the zerofield dynamic susceptibility spectra (imaginary part of the $\chi_{x x}$ element termed $\operatorname{Im}\left(\chi_{x x}\right)$ ) for the three stable configurations within the $[0.5 \mathrm{GHz}-20 \mathrm{GHz}]$ frequency range. 

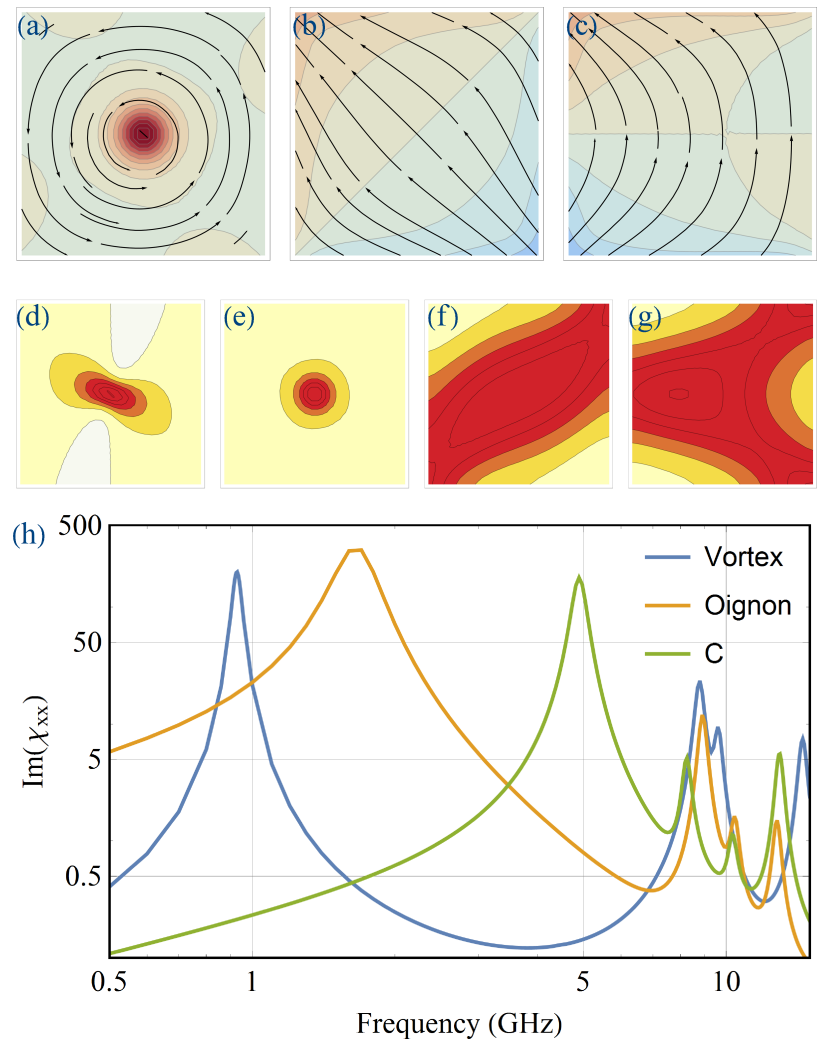

Figure 2. Equilibrium magnetization configurations within the square-shaped element under study; (a) vortex state, (b) onion state and (c) $\mathrm{C}$ state. The color code images the z-component of the static magnetization (positive values in red and negative ones in blue) whereas the arrows represent the in-plane magnetization vector. (h) Dynamic susceptibility spectra (imaginary part of the $\chi_{x x}$ element, $\left.\operatorname{Im}\left(\chi_{x x}(f)\right)\right)$. Maps of the local susceptibility for the vortex state $\left(\operatorname{Im}\left(\chi_{x x}(x, y)\right)\right.$ (d) and $\left.\operatorname{Im}\left(\chi_{+}(x, y)\right),(\mathrm{e})\right)$, the onion state $\left(\operatorname{Im}\left(\chi_{x x}(x, y)\right),(\mathrm{f})\right)$ and the $\mathrm{C}$ state $\left(\operatorname{Im}\left(\chi_{x x}(x, y)\right),(\mathrm{g})\right)$ computed at the resonance frequency of the low-frequency resonances in the $\operatorname{Im}\left(\chi_{x x}\right)$ spectra. The high levels of susceptibility are in red.

A common feature between the three spectra is the appearance of a single low-frequency resonance and multiple resonance lines above $7 \mathrm{GHz}$. For the vortex state in disk-shaped nanostructures, it is well-established that the excitation spectrum consists of a low-frequency vortex translation mode corresponding to a gyrotropic motion of the vortex core as a whole around the element center and high-frequency resonances ascribed to quantized spin-wave modes due to the finite lateral size of the element and essentially spreading outside the vortex core [17]. In what follows, the attention will be focused on the lowest-frequency resonances emerging in the three micromagnetic situations. For our case of a vortex state in a square platelet, the low-frequency resonance located at $\mathrm{f}=0.91 \mathrm{GHz}$ is due to the vortex translation mode as confirmed by the color maps of the imaginary part of the local susceptibility $\left(\operatorname{Im}\left(\chi_{x x}(x, y)\right)\right.$, Fig. 2(d) and, more explicitly, $\operatorname{Im}\left(\chi_{+}(x, y)\right)$ with $\chi_{+}=1 / 2\left[\left(\chi_{x x}+\chi_{y y}\right)+i\left(\chi_{y x}-\chi_{x y}\right)\right]$, Fig. 2(e)) computed at the resonance frequency and showing a high-level of dynamic susceptibility within the vor- tex core. For the onion state, the broad low-frequency resonance at $\mathrm{f}=1.65 \mathrm{GHz}$ is associated with a high level of magnetic response along the opposite diagonal with respect to the privileged one in the static configuration (Fig. 2(f)). For the C state, the low-frequency resonance appears at $\mathrm{f}=4.9 \mathrm{GHz}$. This resonance is due to a high level of magnetic response inside a Y-shape area with the $\mathrm{x}$-axis as the symmetry axis (Fig. 2(g)).

\subsection{Ordered assemblies of nanoplatelets}

\subsubsection{Initialization of the magnetization configuration}

(a)

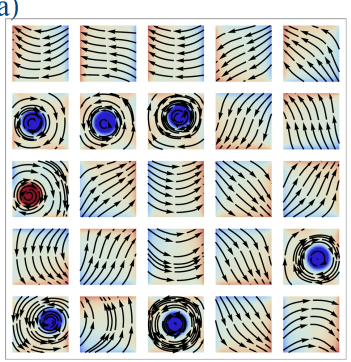

(c)

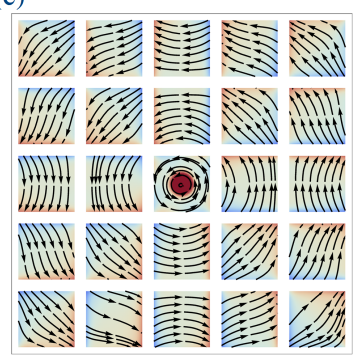

(b)

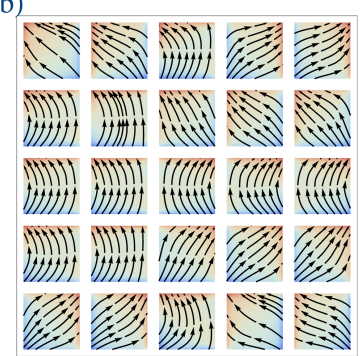

(d)

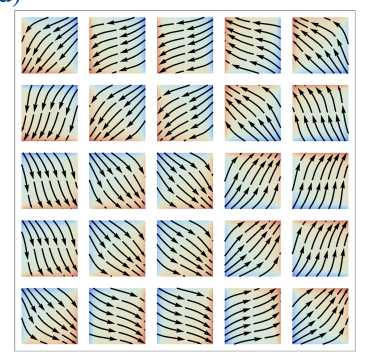

Figure 3. Equilibrium magnetization configurations resulting from various initial magnetization configurations. (a) random configuration, (b) uniform magnetization oriented along a platelet side (y-axis) and centered (c) and off-centered (d) orthoradial magnetization configurations. The geometrical parameters are: $L=90 \mathrm{~nm}, L_{z}=12 \mathrm{~nm}$ and $\delta=15 \mathrm{~nm}$. The color code and the arrows meaning are the same as in Fig. 2.

Let us move on the assemblies of ordered nanoplatelets. The research of an equilibrium micromagnetic configuration requires to start from an initial configuration. The legitim questions from there are how to choose this initial state and what is the impact of this state on the final result. This issue is of prime importance when the micromagnetic simulations are conducted on multiple interacting magnetic objects as in-depth discussed for the search of magnetic ground state in artificial spin ices [8]. To illustrate the effect of this starting state, various initial magnetization configurations have been considered for a square array of nanoplatelets; a random configuration, a uniform configuration with the magnetization vector oriented along a nanoplatelet side (y-axis), centered and off-centered orthoradial magnetization configurations. The orthoradial direction for a given nanoplatelet is defined as the cross product between the position vector at the center of the nanoplatelet and the normal of the 


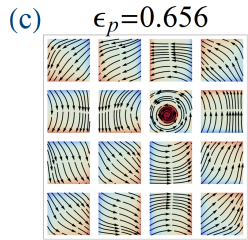

(e)

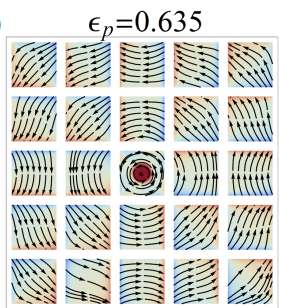

(f)

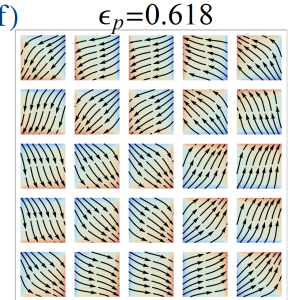

Figure 4. Macro-vortex states for ordered assemblies of nanoplatelets with various square array sizes; $3 \times 3$ ((a) and (b)), $4 \times 4$ ((c) and (d)) and $5 \times 5$ ((e) and (f)). The geometrical parameters are: $L=90 \mathrm{~nm}, L_{z}=12 \mathrm{~nm}$ and $\delta=15 \mathrm{~nm}$. The macro-vortex state has either a vortex core inside a platelet ((a), (c) and (e) configurations) or no vortex core ((b), (d) and (f) configurations). For each configuration, the reduced magnetic energy $\epsilon_{p}$ is provided. The color code and the arrows meaning are the same as in Fig. 2.

array. The nanoplatelets have the following geometrical parameters : $L=90 \mathrm{~nm}$ and $L_{z}=12 \mathrm{~nm}$ with an interplatelet spacing $\delta=15 \mathrm{~nm}$. The array size is fixed at $5 \times 5$. The associated equilibrium magnetization configurations resulting from the micromagnetic simulations are reported in Fig. 3. For the initial random configuration (Fig. 3(a)), a mixing of vortex, onion and $\mathrm{C}$ states is observed within the platelets. In addition, some platelets support an S state (in-plane magnetization along the S-like shape) which is not a stable state for isolated platelets of the same size. The presence of S state reflects the dipolar coupling between the platelets and locally favors the flux closure.

Such magnetic flux arrangements have been experimentally observed using electron holography in FeNi nanoparticle chains [18] and Fe nanocubes [19]. For the uniform intial state (Fig. 3(b)), onion, $\mathrm{C}$ and $\mathrm{S}$ states are stabilized within the platelets. No vortex state is revealed. For the orthoradial initial configurations, the equilibrium magnetization configurations correspond to flux closure patterns with a spiraling in-plane magnetization spreading over the whole platelet array. These configurations are termed macro-vortex states. For a centered orthoradial initial configuration (Fig. 3(c)), a vortex core appears at the central nanoplatelet of the array whereas no vortex core exists for an off-centered initial configuration (Fig. 3(d)). In the last case, the macro-vortex configuration bears some similarities with the local vortex states evidenced in artificial spin ice systems with elongated single-domain elements [20]. To compare the energy between the different configurations, the reduced magnetic energy per platelet is defined as $\epsilon_{p}=\epsilon_{N} /\left(N \epsilon_{v}\right)$, where $\epsilon_{N}$ is the magnetic energy of the array with $\mathrm{N}$ platelets. The above-described equilibrium states have the following energies: $\epsilon_{p}=0.759,0.697$, 0.635 and 0.618 obtained from the random, uniform, centered orthoradial and off-centered orthoradial initial configurations, respectively. It should be remarked that $\epsilon_{p}$ is always lower than unity meaning that the dipolar coupling allows to reduce the magnetic energy with respect of a collection of noninteracting platelets. In addition, the offcentered macro-vortex state has the lowest energy. From the experimental point of view, the orthoradial magnetization configurations can be carried out by applying an in-plane rotating magnetic field and then reducing its amplitude down to zero.

In the present work, the purpose is to investigate the mi- crowave susceptibility spectra associated with flux closure patterns and to evidence the conditions for appearing a low-frequency resonance (below $1 \mathrm{GHz}$ ).

\subsubsection{Flux closure magnetic configurations}

Figure 4 shows the computed lowest-energy equilibrium states with a flux closure pattern for various array sizes $(N=3 \times 3,4 \times 4$, and $5 \times 5)$. The platelet size and the platelet thickness are fixed at $L=90 \mathrm{~nm}$ and $L_{z}=12 \mathrm{~nm}$, respectively. The interplatelet spacing $\delta$ is equal to $15 \mathrm{~nm}$. The equilibrium magnetization configurations correspond to macro-vortex states. Several comments can be drawn. (i) The macro-vortex configurations can be either centered (Figs. 4(a), 4(d) and 4(e)) or off-centered (Figs. 4(b), 4(c) and 4(f). The centered macro-vortex state with a vortex core (resp. without a vortex core) appears in arrays with an odd (resp. even) number of cells. (ii) For a given array size, the lowest-energy state is always the magnetization configuration without a vortex core but the energy differences between the configurations with and without a vortex core are weak (see Fig. 4).

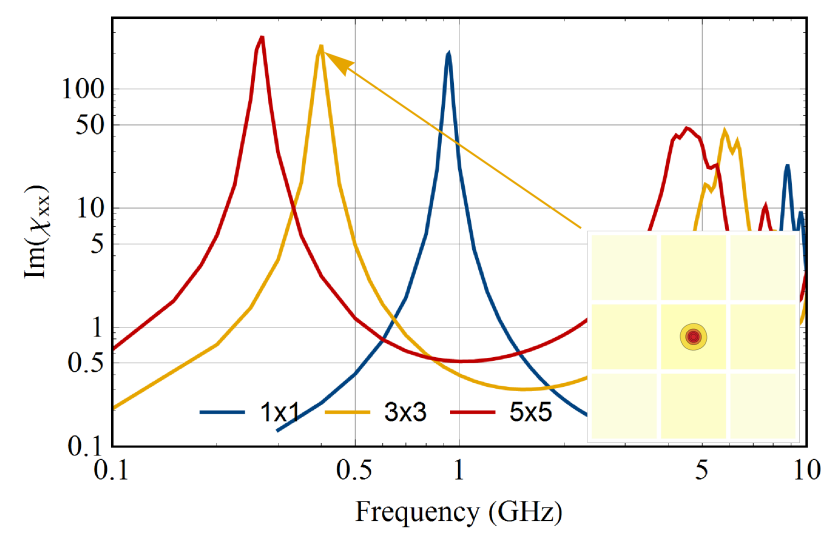

Figure 5. Ordered arrays of nanoplatelets with a centered macrovortex state and a vortex core. Array size dependence of the $\operatorname{Im}\left(\chi_{x x}\right)$ spectrum. The interplatelet spacing is $\delta=3 \mathrm{~nm}$. The inset maps the local susceptibility $\left(\operatorname{Im}\left(\chi_{+}(x, y)\right)\right.$ of the $3 \times 3$ array.

Figure 5 and 6 displays the $\operatorname{Im}\left(\chi_{x x}\right)$ spectra of magnetization configurations with a centered macro-vortex state 


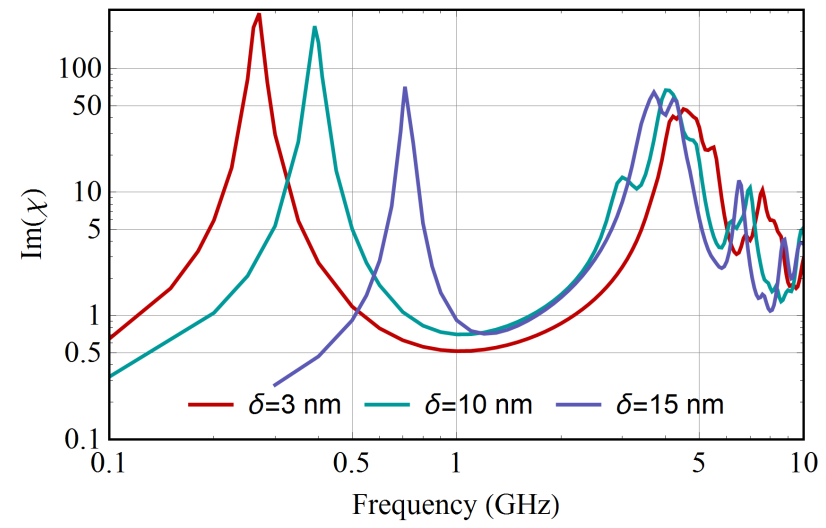

Figure 6. Ordered arrays of nanoplatelets with a centered macrovortex state and a vortex core. Effect of the interplatelet spacing on the $\operatorname{Im}\left(\chi_{x x}\right)$ spectrum. The array size is fixed at $5 \times 5$.

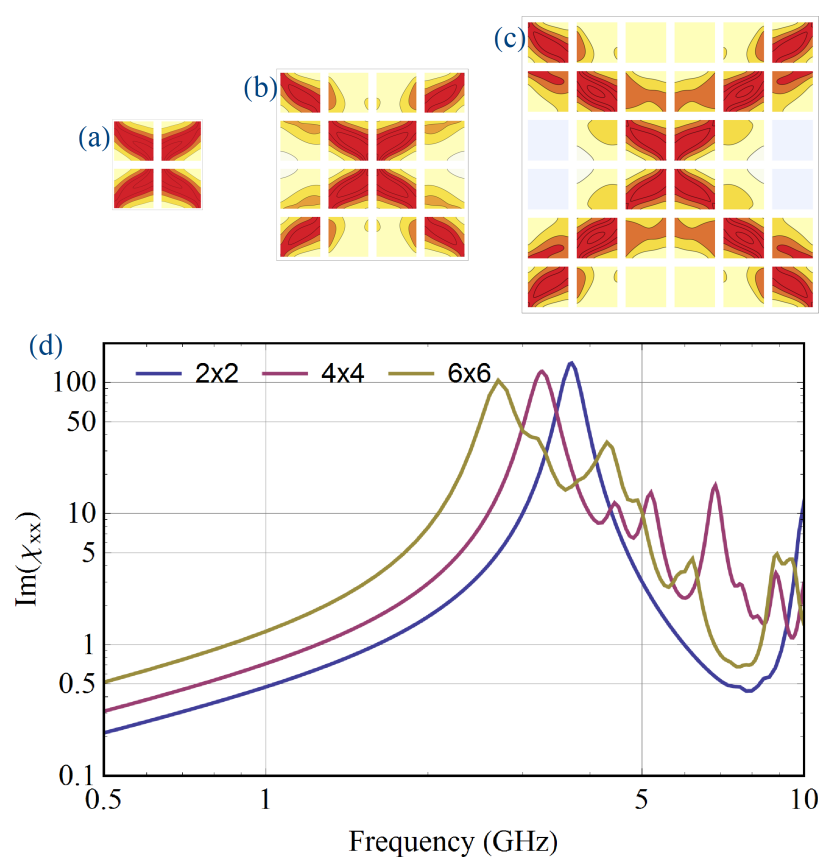

Figure 7. Ordered arrays of nanoplatelets with a macro-vortex state but no vortex core. (d) Array size dependence of the $\operatorname{Im}\left(\chi_{x x}\right)$ spectrum. The interplatelet spacing is $\delta=3 \mathrm{~nm}$. Maps of the local susceptibility (imaginary part) associated with the low-frequency resonance for arrays of size $2 \times 2$ (a), $4 \times 4$ (b) and $6 \times 6$ (c). The color code is the same as in Fig. 2.

with a vortex core for various array sizes (Figure 5) and for various interplatelet spacings (Figure 6). These spectra are mainly characterized by an high-amplitude and lowfrequency resonance (below $1 \mathrm{GHz}$ ). The local susceptibility map reveals that this resonance is localized within the vortex core at the array center and is associated with the macro-vortex translation mode by analogy to the single platelet case (inset in Fig.5). Increasing the size array for a given interplatelet spacing, $\delta=3 \mathrm{~nm}$, (Fig. 5) leads to a lowering of the resonance frequency of the macro-vortex translation mode. This behavior is consistent with the one of a unique platelet with the increasing platelet size. Ris- ing the interplatelet spacing for an array size fixed at $5 \times 5$ (Fig. 6), results in a frequency shift of the resonance frequency of the macro-vortex translation mode towards the high frequencies as a consequence of reducing the dipolar interaction between the platelets. As a result, the resonance frequency of this low-frequency mode can be typically tuned between $200 \mathrm{MHz}$ and $1 \mathrm{GHz}$.

The $\operatorname{Im}\left(\chi_{x x}\right)$ spectra of magnetization configurations with a centered macro-vortex state without a vortex core for various array sizes are reported in Figure $7(\delta=3 \mathrm{~nm})$. In each case, a unique high-amplitude resonance exists as well but the resonance frequency is located above $2 \mathrm{GHz}$. The local susceptibility maps point out that this resonance is due to a mode extended over the whole array with a fourfold symmetry. Increasing the array size leads to a decrease of the resonance frequency.

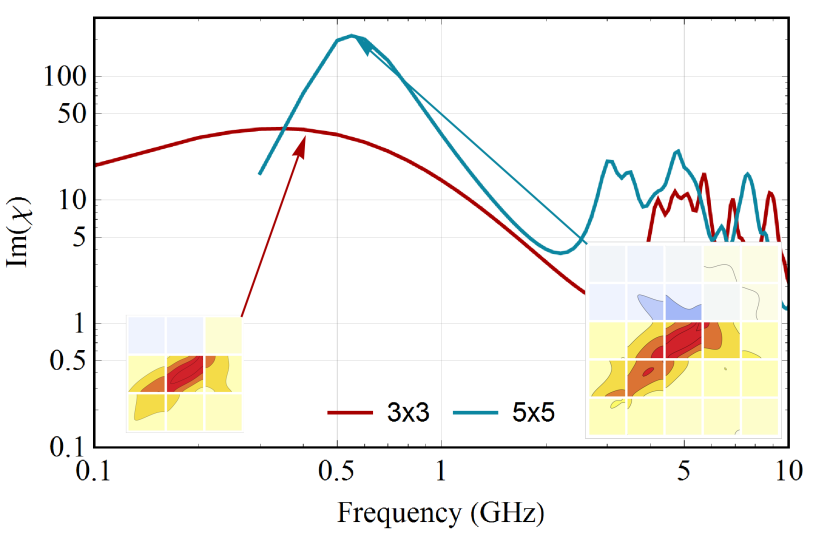

Figure 8. Ordered arrays of nanoplatelets with an off-centered macro-vortex state without a vortex core. $\operatorname{Im} \chi_{x x}$ spectra for two array sizes $3 \times 3$ and $5 \times 5$. Regime of strong dipolar coupling $(\delta=$ $3 \mathrm{~nm}$ ). The insets correspond to the local susceptibility maps (imaginary part) of the low-frequency resonance for the two array sizes. The color code is the same as in Fig. 2.

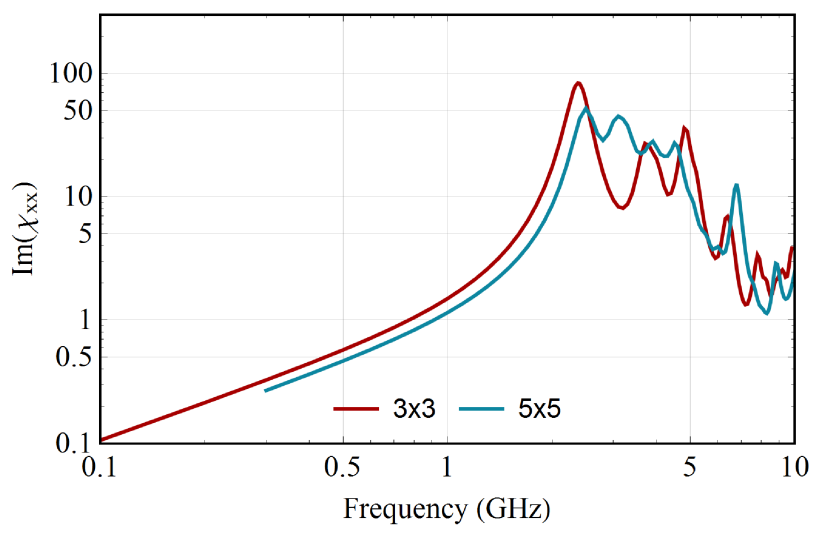

Figure 9. Ordered arrays of nanoplatelets with an off-centered macro-vortex state without a vortex core. $\operatorname{Im} \chi_{x x}$ spectra for two array sizes $3 \times 3$ and $5 \times 5$. Regime of weak dipolar coupling $(\delta=$ $15 \mathrm{~nm})$.

Figure 8 shows the $\operatorname{Im}\left(\chi_{x x}\right)$ spectra for magnetization configurations with an off-centered macro-vortex state 
without a vortex core. Interestingly, the presence of a lowfrequency resonance (below $1 \mathrm{GHz}$ ) depends on the dipolar coupling strength. For a strong dipolar coupling $(\delta=3$ $\mathrm{nm})$, a low-frequency response exists with a marked resonant shape for a large enough array size (Fig. 8). It should be noted that the amplitude of this resonance is high for this array size. The local susceptibility map reveals that this resonance arises from a cooperative mode extending over several platelets. For the regime of a weak dipolar coupling ( $\delta=15 \mathrm{~nm}$ ), no low-frequency response is observed whatever the array size (Fig. 9). It should be remarked that resonance lines assigned to nonuniform collective excitations within arrays have been reported for stadium shaped nanoelements in a square array [9]. However, such nanoelements are uniformly magnetized by an in-plane bias magnetic field in contrast to the nanoplatelets considered in this work.
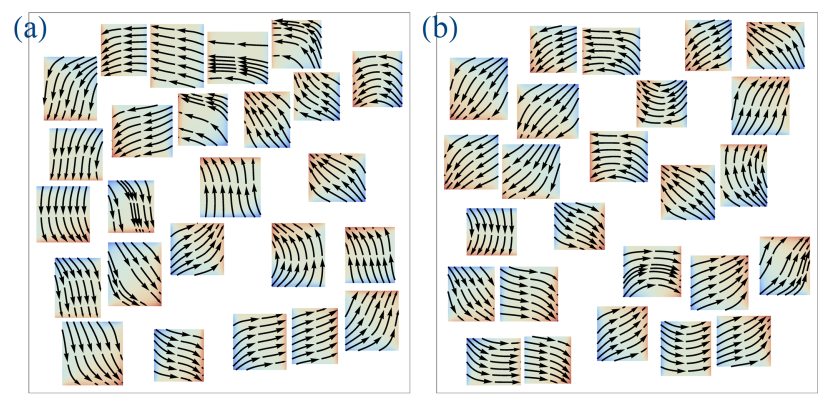

(c)
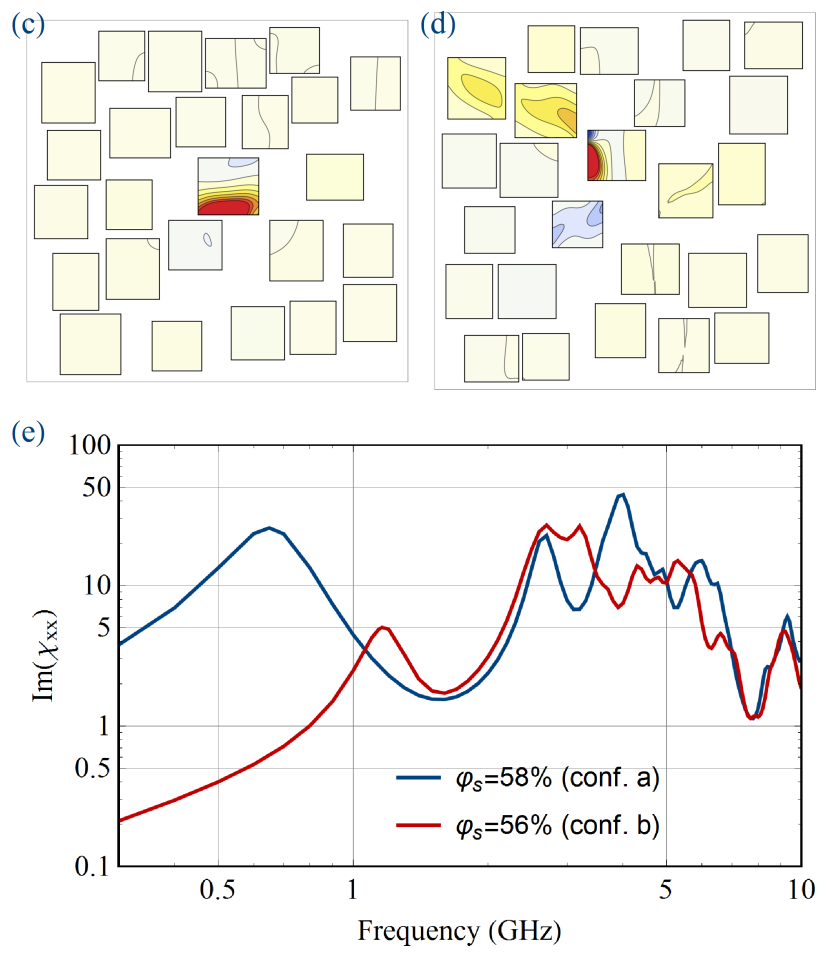

Figure 10. Disordered assemblies of nanoplatelets numerically generated with a surface content $\varphi_{s}=58 \%$ (a) and $\varphi_{s}=56 \%$ (b). (e) $\operatorname{Im}\left(\chi_{x x}\right)$ spectra for the two above described configurations. Maps of the local susceptibility (imaginary part) associated with the low-frequency resonance for the $\varphi_{s}=58 \%$ (c) and $\varphi_{s}=56 \%$ (d) configurations. The color code is the same as in Fig.2.

\subsection{Disordered assemblies of nanoplatelets}

As an example, the case of disordered aggregates formed by 25 rectangular platelets are considered. The platelet thickness is fixed at $L_{z}=4 \mathrm{~nm}$. The lateral size distribution of nanopatelets and their relative positions were generated according to the process described in Sec.2.1. The surface content of nanoplatelets, $\varphi_{s}$ is changed by compacting them along the $\mathrm{x}$ and $\mathrm{y}$-axes.

The results coming from two independent draws corre-

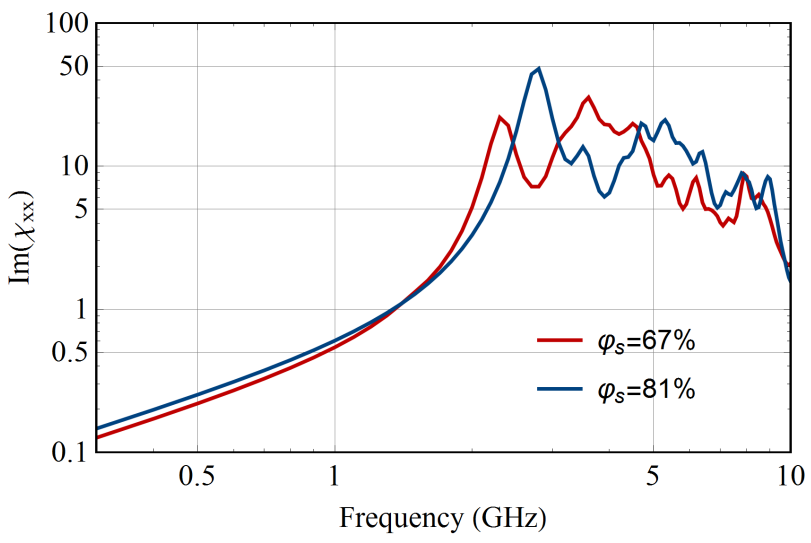

Figure 11. Disordered assemblies of nanoplatelets. $\operatorname{Im}\left(\chi_{x x}\right)$ spectra for two high surface contents $\varphi_{s}=67 \%$ and $\varphi_{s}=81 \%$.

sponding to $\varphi_{s}=58 \%$ and $\varphi_{s}=56 \%$ are presented in Fig. 10. In each case, the starting state is an orthoradial magnetization configuration. The two equilibrium magnetization configurations (case $\varphi_{s}=58 \%$, Fig. 10(a) and case $\varphi_{s}=56 \%$, Fig. 10(b)) are flux closure states essentially mixing $\mathrm{S}$ states, $\mathrm{C}$ states and onion states at the level of individual nanoplatelets. Figure 10(e) shows the associated $\operatorname{Im}\left(\chi_{x x}\right)$ spectra. These ones reveal the existence of a low-frequency resonance at $0.65 \mathrm{GHz}$ for $\varphi_{s}=58 \%$ and at $1.16 \mathrm{GHz}$ for $\varphi_{s}=56 \%$ but with a weaker amplitude with respect to the case of the periodic arrays. The resonance lines arise from a mode essentially localized inside one nanoplatelet (case $\varphi_{s}=58 \%$, Fig. 10(c)) or extended over three nanoplatelets (case $\varphi_{s}=56 \%$, Fig. 10(d)). Despite a quite similar surface content, these two assemblies of nanoplatelets lead to distinct feartures of the low-frequency resonance in terms of resonance position, resonance linewidth and line amplitude. Let us now consider the case of higher surface contents. The $\operatorname{Im}\left(\chi_{x x}\right)$ spectra for two disordered aggegates with $\varphi_{s}=67 \%$ and for $\varphi_{s}=81 \%$ are displayed in Fig. 11. A striking feature is the absence of a low-frequency resonance for these very dense composite media. These results highlight the impact of disorder on the dynamical behavior of dipolar coupled nanoplatelets and the difficulty to control the lowfrequency part of the susceptibility spectra.

\section{Summary and perspectives}

These numerical results illustrate the large variety of existing microwave susceptibility spectra even for the idealistic case of assemblies of thin soft platelets with a regu- 
lar shape coupled by dipolar interactions. Restricting to magnetization configurations with a flux closure pattern at the array scale, a high-amplitude and low-frequency resonance (below $1 \mathrm{GHz}$ ) exists in ordered arrays supporting a macro-vortex state with a vortex core but also without a vortex core for the regime of strong dipolar coupling. For the disordered aggregates, a low-frequency absorption line (between $0.5 \mathrm{GHz}$ and $2 \mathrm{GHz}$ ) emerges as well but with a weaker amplitude. This resonance appears in flux closure states and are associated with a cooperative mode spreading over a few platelets. Such resonances disappear for higher surface contents. These preliminary results evidence the strong impact of disorder on the susceptibility spectra.

These micromagnetic simulations of interacting magnetic nanostructures can be viewed as a first step to get a better understanding of the microwave magnetic response of real systems. To go further, several points will have to be taken into account. First, the magnetic history of the sample plays a critical role and must be closely reproduced in the demagnetization protocols used in the micromagnetic simulations. Second, the presence of defects, as well as the shape and size distributions of nanoparticles can affect the equilibrium magnetizations configurations rending difficult to control and predict the microwave magnetic behavior. Introduction of information on the nanocomposite morphology in the micromagnetic simulations is needed. Third, clustering effect occurs in magnetic nanocomposites with a significative volume content of particles. The micromagnetic simulations will have to address this issue. This overall challenge is ambitious but in phase with the perspective of using arrangements of 2D or 3D nanoparticles in future devices.

\section{References}

[1] C.A. Ross, Annual Review of Materials Research 31, 203 (2001)

[2] A. Imre, G. Csaba, L. Ji, A. Orlov, G.H. Berstein, W. Porod, Science 311, 205 (2006)

[3] N. Jones, Nature 472, 22 (2011)
[4] Q.A. Pankhurst, J. Connolly, S.K. Jones, J. Dobson, J. Phys. D Appl. Phys. 36, 167 (2003)

[5] T.H. Kim, E.Y. Jang, N.J. Lee, D.J. Choi, K.J. Jang, J.S. Choi, S.H. Moon, J. Cheon, Nanoletters 9, 2229 (2009)

[6] J.I. Martin, J. Nogues, K. Liu, J.L. Vicent, I.L. Schuller, J. Magn. Magn. Mat. 256, 449 (2003)

[7] C. Nisoli, R. Moessner, P. Schiffer, Rev. Mod. Phys. 85, 1473 (2013)

[8] L.J. Heyderman, R.L. Stamps, J. Phys: Condens. Matter 25, 363201 (2013)

[9] V.V. Kruglyak, P.S. Keatley, A. Neudert, R.J. Hicken, J.R. Childress, J.A. Katine, Phys. Rev. Lett. 104, 027201 (2010)

[10] S. Gliga, A. Kakay, R. Hertel, O.G. Heinonen, Phys. Rev. Lett. 110, 117205 (2013)

[11] R. Ramprasad, P. Zurcher, M. Petras, M. Miller, P. Renaud, J. Appl. Phys. 96, 519 (2004)

[12] K.N. Rozanov, M.Y. Koledintseva, J.L. Drewniak, J. Magn. Magn. Mater. 324, 1063 (2012)

[13] W.T. Doyle, Phys. Rev. B 39, 9852 (1989)

[14] S. Labbe, P.Y. Bertin, J. Magn. Magn. Mater. 206, 93 (1999)

[15] F. Boust, N. Vukadinovic, Phys. Rev. B 70, 172408 (2004)

[16] N. Vukadinovic, F. Boust, Phys. Rev. B 75, 014420 (2007)

[17] L.M. Lacroix, S. Luchaize, F. Hue, C. Gatel, T. Blon, R.P. Tan, J. Carrey, B. Warot-Fonrose, B. Chaudret, Nano. Lett. 12, 3245 (2012)

[18] C. Gatel, F.J. Bonilla, A. Meffre, E. Snoeck, L.M.L. B. Warot-Fonrose, B. Chaudret, T. Blon, Nano. Lett. 15, 6952 (2015)

[19] E. Snoeck, C. Gatel, L.M. Lacroix, T. Blon, S. Lachaize, J. Carrey, M. Respaud, Nano. Lett. 8, 4293 (2008)

[20] A.G. Gurevich, G.A. Melkov, Magnetization Oscillations and Waves (CRC Press, Boca Raton, Florida, 1996) 